\title{
Molecular determinants of resistance in Escherichia coli-ST131 isolated blood stream infections
}

\author{
Adriana Hristea ${ }^{1,2^{*}}$, Raluca Jipa ${ }^{2}$, Ioana D Olaru², Cristina Popescu ${ }^{1,2}$, Victoria Aramă ${ }^{1,2}$, Ruxandra Moroti ${ }^{1,2}$, \\ Oana Streinu-Cercel ${ }^{1,2}$ \\ From The 9th Edition of the Scientific Days of the National Institute for Infectious Diseases Prof Dr Matei Bals \\ Bucharest, Romania. 23-25 October 2013
}

\section{Background}

During the past years an E coli clonal lineage ST131 has emerged explosively, causing predominantly communityonset antimicrobial resistant infections. E coli ST131 has been shown to harbor a number of virulence and resistance genes and is now recognized for its ability to cause potentially severe infections in many parts of the developing world, implying public health measures in attempt to control infection.

\section{Methods}

Eighty-five drug-resistant $E$ coli isolates from patients with blood stream infections (BSI) were screened by a ST131-clone allele-specific PCR for the pabB gene and analyzed for genes encoding ESBL by PCR and sequencing. Integrons were detected by PCR targeting the integrase gene. Gene cassette PCR was conducted on all class 1 integrase-positive isolates. BSI were classified according to the setting of infection: community acquired (CA), healthcare-associated (HCA), and hospital acquired (HA).

\section{Results}

The sources of E coli BSI, were: urinary in 50 (59\%), gastrointestinal in $9(11 \%)$, respiratory in $4(5 \%)$, other (including skin and soft tissue) in $3(3 \%)$, unknown in 19 (22\%) patients. By pabB PCR, we identified 22 (26\%) $E$ coli isolates belonging to the O25b-ST131 clonal lineage. Among 22 ST131 E coli isolates, 6 (27\%) were from CA, $11(50 \%)$ were from HCA, and $5(23 \%)$ were from HA infections, while among 61 non-ST $131 \mathrm{E}$ coli isolates, $30(49 \%)$ were from CA, 14 (23\%) were from HCA, and 17 (28\%) from HA infections ( $\mathrm{p}=0.053)$. ESBL genes were found in 20 (91\%) ST131 and 13 (21\%) non-ST131 E coli isolates $(\mathrm{p}<0.0001, \mathrm{OR}=38.46$ [95\%CI: 7.95-186.06]). The ST131 isolates carried $b l a_{\mathrm{CTX}-\mathrm{M}}$ in $18(82 \%), b l a_{\text {TEM-1 }}$ in $6(27 \%)$ and $b l a_{\mathrm{OXA}-1}$ in $18(82 \%)$. Class I integrons were identified in 11 (50\%) ST131-producing E coli strains and 36 (57\%) non-ST131 E coli strains ( $\mathrm{p}=0.623, \mathrm{OR}=0.750$ [95\%CI: 0.283-1.9850]). Resistance to trimethoprim/sulfamethoxazole was found in 11 (50\%) ST131 E coli isolates and in $32(59 \%)$ non-ST131 E coli strains (p=0.610), while resistance to both fluoroquinolones and aminoglycosides was identified in 18 (82\%) ST131 E coli isolates compared to $10(16 \%)$ non-ST131 E coli strains $(\mathrm{p}<0.001)$. Among patients with BSI caused by resistant $E$ coli, the in-hospital mortality was similar in patients infected with strains carrying the ST131 clonal lineage (3; 14\%) versus non ST131 $(10 ; 17 \%)(\mathrm{p}=0.921)$.

\section{Conclusion}

In our study group, BSI caused by $E$ coli ST131 were most likely to be hospital rather than community associated. We found more ESBL genes in ST131 E coli isolates, while class I integrons were present in both groups in similar rates.

\section{Authors' details}

${ }^{1}$ Carol Davila University of Medicine and Pharmacy, Bucharest, Romania. ${ }^{2}$ National Institute for Infectious Diseases "Prof. Dr. Matei Balş", Bucharest, Romania.

Published: 16 December 2013

doi:10.1186/1471-2334-13-S1-015

Cite this article as: Hristea et al:: Molecular determinants of resistance in Escherichia coli-ST131 isolated blood stream infections. BMC Infectious Diseases 2013 13(Suppl 1):015.

* Correspondence: adriana_hristea@yahoo.com

${ }^{1}$ Carol Davila University of Medicine and Pharmacy, Bucharest, Romania

Full list of author information is available at the end of the article

(c) 2013 Hristea et al; licensee BioMed Central Ltd. This is an Open Access article distributed under the terms of the Creative Commons Attribution License (http://creativecommons.org/licenses/by/2.0), which permits unrestricted use, distribution, and reproduction in any medium, provided the original work is properly cited. 